Table 2. Benchmark

\begin{tabular}{lc}
\hline Invasion & KP \\
\hline Giardia & 2,8 \\
Clonorchis & 1,7 \\
Toxocara & 1,5 \\
Ascaris & 3,1 \\
\hline
\end{tabular}

A positive result of the study is considered to be $K P>1$, which tells about the infestation of the body or that the invasion took place in the past. Given that parasitic infestations with increased levels of eosinophils, we studied this indicator in children. The mean value of eosinophils in children with the identified antibody is $2.32 \%$, and in children with negative results of $1.2 \%$. According to the results of IFA, children were administered anthelmintic therapy drugs: neosol, mebendazol, biltricid, praziquantel.

Conclusion. $1 / 3$ of children, observed with a diagnosis of chronic gastroduodenitis and immunodeficiency States identified antibodies to clonorchis, Giardia, Toxocara, roundworm. Children with KP >1.1 to 1.2, which may indicate infestation, it is necessary to appoint additional examination methods such as PCR, stool for detection of cysts of Giardia, Toxocara, clonorchis and others. $15.3 \%$ of cases according to the results of IFA were prescribed treatment.

\title{
Literature
}

1. Medical Parasitology and parasitic diseases. № 2, 2011. p. 47.

2. Kharchenko G. A. Intestinal infections in children of early age.// Phoenix. 2007. P. 257-263.

3. Egorov A. M., A. P. Osipov, B. B. Dzantiev Theory and practice of enzyme immunoassay.//GEOTAR-Media. 2007. S. 308.

DOI: 10.22448/AMJ.2016.15-16.28-31

\section{UDC 616.727.13; 616.72-001.6-089.85 \\ FIXATION OF ACUTE ACROMIOCLAVICULAR JOINT INJURIES (ROCKWOOD II-VI) WITH MODELED KIRSCHNER WIRE AND CORTICAL SCREW}

\section{Borozda I. V., Danilov M. A.}

\section{Amur State Medical Academy, Blagoveshchensk, Russia}

Introduction. Dislocation of the acromial extremity of the clavicle is a fairly common injury of the locomotor system, constituting from 3\%-15\% to $19 \%$ of the total number of dislocations and being the third most common dislocation after the shoulder and forearm. Those most frequently affected by these injuries are young, able-bodied people, who lead an active lifestyle and engage in sport.

The complex biomechanical relationships in the acromioclavicular joints of humans, when the biasing force is caused by the action of muscles, provoke clavicle dislocation, and ruptured capsuleligamentous apparatus is unconducive to the maintenance of stability. All of this leads to difficulties in treatment that are naturally accompanied by a high rate of complications and relapse. To determine the optimal method of treatment, the correct biomechanical assessment of the injury to the ligamentous apparatus of the clavicle is vital, which is impossible to determine without the particular role of one or other ligament in retaining the clavicle in the correct anatomical position.

At the same time, in the available scientific literature we analyzed, there is no consensus on the degree of importance of the elements of the ligament apparatus of the acromioclavicular joint when dislocation occurs. Thus, some authors have noted the occurrence of dislocation following damage to the acromioclavicular ligament alone. Others believe that, for the occurrence of a complete dislocation, damage to both the acromioclavicular and the clavicular-coracoid ligament is necessary. However, all authors agree that damage to the acromioclavicular ligament leads to a particular violation of the relationship of the articular surfaces in the acromioclavicular joint. Thus, understanding the role of the ligamentous apparatus during rupture to the acromioclavicular joint expressed contradictions are traced. This provides a basis for the further biomechanical study of the acromioclavicular joint through the determination of the optimal location for the application and construction of the retainer for its stabilization. For the treatment of injury to the acromioclavicular, joint both conservative and operational methods are used. Different types of splints, bandages and casts came into widespread use throughout the last century. However, the imperfection of their construction, awkwardness, 
discomfort and high recurrence rate significantly reduced the frequency of their use. Surgical methods are more convenient for patients in terms of post-operative treatment and social adaptation.

Nowadays, there exist more than 300 techniques of surgical treatment for rupture of the acromioclavicular joint, including plastic acromial extremity of the clavicle (AEC) ligaments, splinting, and perosseous osteosynthesis.

The most high-tech AEC treatment methods involve restoring the clavicular-coracoid ligament through the construction of rigid threads, for example Endobutton, including the use of endoscopic techniques. The minimally invasive acromioclavicular reconstruction method is also widespread.

However, despite the use of these expensive metal and surgical approaches, the number of postoperative complications remains high, so Lin et al. performed a study that found that hamate plates use for the recovery of AEC lead to impingement syndrome in $37.5 \%$ and to damage to the rotator cuff shoulder in $15 \%$ of patients following hamate plate removal, which resulted in significantly limited motion in the shoulder joint. Thus, the development of new methods of surgical treatment for dislocation of the acromial extremity of the clavicle is topical as well as socially and economically justified. The aim of this work was to improve the results of treatment for patients with traumatic damage to the acromioclavicular joint and dislocation of the acromial extremity of the clavicle.

Materials and methods. Biomechanical testing was performed on 43 human cadavers of both sexes ( 31 men, 12 women) who had died from somatic pathology. It was performed in three stages. All manipulations of cadavers were carried out in accordance with permission from the Ethics Committee of the Amur State Medical Academy. In the first stage, the change in the relationship between the articular surfaces of the clavicle and the acromial process of the blade at the sequential intersection of the elements of musculo-capsular ligament was studied to determine the significance of the elements of the ligamentous apparatus in stabilizing the acromioclavicular joint.

The second stage involved the evaluation of the biomechanical strength parameters for each of the ligaments of the acromioclavicular joint.

The third stage entailed a study of the stability of the acromioclavicular joint in the condition of osteosynthesis by the proposed device.

The clinical study was performed in accordance with the Declaration of Helsinki (1964), as well as with permission from the Ethics Committee of the Amur State Medical Academy and included an analysis of the results of the treatment of 154 patients with closed injuries to the acromioclavicular joint, who were hospitalized at Amur Regional Hospital (Blagoveshchensk) during the period between 2000 and 2013. All patients were divided into a study group (34 patients) and a clinical comparison group (120 patients). Treatment was carried out with the informed agreement of the patients in a drawn-up protocol for each person $(n=154)$.

The absence of significant differences between patients by sex, age and circumstances of injury in the test groups indicated that they were uniformly formed. The main group (the prospective study) included patients operated by our methodology with the use of a modeled $1.8 \mathrm{~mm}$ Kirschner wire and $3.5 \mathrm{~mm}$ screws (Russian Federation Priority Reference No 201110773).

The clinical comparison group (retrospective study) consisted of patients who received treatment in the form of different types of submersible constructions: Lee hook $(n=76)$, Kirschner wire $(n=25)$, hamate plate $(n=19)$.

The conventional preoperative preparation was followed. The operation was performed at 2-3 days of receipt. As the anesthetic technique, endotracheal anesthesic was used. Operational line access of $4 \mathrm{~cm}$ in length was performed directly above the acromioclavicular joint. Damaged meniscus was removed. Usually, the construction was produced during the operation, based on the individual patient's anatomy, for which a Kirschner wire was bentwith the help of pliers. After installing the construction and the removal of the dislocation, in the clavicle using a drill of diameter $2.8 \mathrm{~mm}$ was formed a channel in the sagittal plane, in the front-toback direction to enable the introduction of a $3.5 \mathrm{~mm}$ screw. Carving was cut in the channel. After the introduction of the screw and control for hemostasis, the wound was sutured in layers. In the early postoperative period, debridement was performed with antiseptic solutions, and the sutures were removed 11-12 days post-surgery.

The fixation of the shoulder was carried out using a soft bandage. Then the patient was discharged to outpatient treatment with a recommendation to limit physical activity within 1 month after surgery. In this period, physiotherapy exercises were performed to develop motion in the shoulder joint. After this time, the patient was discharged to work. Removal of construction was carried out 4 months after the primary surgery.

The result of the treatment was monitored in dynamics and measured in points. The immediate results were assessed using the scale of Constant and Murley (1987) 4 months post-surgery (prior to removal of the construction).

A statistical analysis of the results was performed via standard methods of mathematical statistics using the software packages Excel and Statistica v.8.o. To assess the sample, the average (M) and the error of the 
mean $(\mathrm{m})$ were calculated, and the significance of the differences between the mean values were assessed using a parametric student's t-test. Differences were considered significant at $P<0.05$.

Results. Over the sequential intersection of the musculo-capsular ligament apparatus of the acromioclavicular joint (Stage I of the biomechanical study), the following data were obtained: over the clipping from the clavicle M. deltoideus and $M$. trapezius, discongruence in the acromioclavicular joint did not occur, however, it appeared at a bias instability of the clavicle anteriorly and posteriorly. Additional crossing of the acromioclavicular ligament led to the expansion of the joint gap to $(0.73 \pm 0.02) \mathrm{cm}$, with the displacement of the clavicle appearing up to $(1.01 \pm 0.02) \mathrm{cm}$. Subsequent crossing of the clavicular-coracoid ligament led to the increased displacement of the clavicle up to $(1.57 \pm 0.03) \mathrm{cm}$ and posteriorly to $(2.12 \pm 0.04) \mathrm{cm}$. The isolated intersection of the clavicular-coracoid ligament caused a downward shift in the coracoid process of the blade, while irregularities in the relationships between the acromioclavicular joint were not mentioned.

At the same time, isolated intersection of the acromioclavicular ligament and joint capsule led to the expansion of the joint gap to $(0.69 \pm 0.03) \mathrm{cm}$, with the occurrence of a clavicle upward shift at $(0.97 \pm 0.07) \mathrm{cm}$.

Investigation of the strength characteristics of the acromioclavicular ligament (II stage) was carried out using the original biomechanical stand.

We found that the amount of force that led to the rupture of the ligament was $[(893.00 \pm 1.45)(H)]$, with the maximum amount of displacement of the acromial extremity of the clavicle before the rupture being (4.00 $\pm 0.18) \mathrm{mm}$.

During the III experimental phase, studies have found that the critical strain of the proposed construction occurs with the $[(116.00 \pm 1.15)(H)]$ load, with the maximum amount of displacement of the acromial extremity of the clavicle before the rupture being $[(4.50 \pm 0.02) \mathrm{mm}$. While comparing the results of the treatment in a group of clinical comparisons and basic group using the scale of Constant and Murley (1987)[24], the following parameters were evaluated (Table 1). These data confirmed the effectiveness of the developed technique. So, at the end of treatment ( 4 months after the initial surgery), the average was 95.90 points, which corresponded to an excellent result. In the comparison group, the index was: over osteosynthesis with Lee hook-89.70 points, intradermal fixation-91.10 points, osteosynthesis with hamate plate-93.60 points.

To fix the hands of the main group of patients after the operation, a Dezo soft bandage was used. In the clinical comparison group, for external fixation was used a Smirnov-Weinstein cast-103 (85.9\%) cases, a Dezo cast-15 (12.5\%) and a Turner cast-2 cases (1.6\%). The overall hospital stay for the study group was (13.00 \pm $0.71)$, while the postoperative hospital stay was $(8.94 \pm 0.48)$. The same indexes were received for the comparison group: osteosynthesis with Lee hook $(15.10 \pm 0.46)$ and $(11.10 \pm 0.39)(P<0.001)$, intradermal fixation $(11.70 \pm 0.84)$ and $(8.56 \pm 0.91)(P>0.05)$, and osteosynthesis with hamate plate $(12.20 \pm 0.97)$ and $(8.32 \pm$ $0.89)(P>0.05)$.

The duration of temporary disability among the study group (40.40 0.71 ) was 15,11 and 2 days less than when using the Lee hook $\left(55.20 \pm 0.57, \mathrm{P}_{1}<0.001\right)$, fixing with the wires $(51.70 \pm 0.96, P<0.001)$ and using the hamate plate $(42.10 \pm 0.67, \mathrm{P}>0.05)$, which indicated the effectiveness of the proposed method.

The greatest number of complications in patients in the clinical comparison group was observed after the application of the Lee hook [26 (33.9\%)]. Percutaneous fixation by Kirschner wires was accompanied by complications in 12 (48\%) cases, osteosynthesis by hamate plate in $4(21 \%)$ cases.

The complication rate in the study group was 3 cases (8.7\%). In one case, it was a superficial inflammation in the surgical wound, broken by the introduction of antibiotics that, in the end, did not affect the duration of treatment.

Deforming arthrosis of the acromioclavicular joint in the same occurred in one patient. Even though in one case there was a fracture of construction, in our opinion, that was incidental with nonobservance by the patient, who began to perform full physical stress on the shoulder joint in the early postoperative period.

Discussion. In terms of biomechanical experiment, it was revealed that the main role in stabilizing the acromioclavicular joint belongs to the acromioclavicular ligament and joint capsule, in which an over isolated intersection is marked by the complete dissociation (dislocation) of the articular surfaces. The displacement of the clavicle is up $[(1.01 \pm 0.02) \mathrm{cm}]$, and the expansion of joint gap $[(0.73 \pm 0.02) \mathrm{cm}]$. Rupture of the acromioclavicular ligaments and joint capsule occurs at a force [(893.00 \pm 1.45$)(\mathrm{H})]$.

Therefore, the most rational option for surgical treatment is the method of the temporary splinting of the acromioclavicular joint by submerged metallic construction that creates stability compared with this indicator.

An original construction for the osteosynthesis of the acromioclavicular joint (priority reference No. 201110773), with the following qualities: simplicity of manufacture, availability, personality (made in the operating room, depending on the patient's anatomy) is proposed.

Through its use, unlike other metal constructions, used less invasive access $4 \mathrm{~cm}$ length, micromotion in the acromioclavicular joint persists and there is no interponing implant between articular surfaces. In the context of biomechanical experiment, the proposed construction demonstrated the ability to withstand the biasing force of $[(116.00 \pm 1.15)(H)]$, which corresponds to the strength parameters of the acromioclavicu- 
lar-clavicular ligament.

These estimates are the results of treatment of 34 patients in the main group using the scale of Constant and Murley (1987)[24] to confirm the effectiveness of the developed method. At the end of treatment (4 months after surgery), the average score was 95.90 balls, that corresponds to an excellent result, and is no worse than those of the comparison group, which carried out osteosynthesis with a hook plate (93.60 points), fixing with Lee hook (89.70 points), and percutaneous fixation with Kirschner wires (91.10 points).

The application of the proposed method of treatment leads to an increase of the quantity of complications by $12.3 \%$ compared with the hamate plate $(P>0.05), 25.2 \%$-Lee hook fixation $(P<0.01)$ and 39.3\%-Kirschner wires $(P<0.001)$.

The overall hospital stay in the study group was $(13.00 \pm 0.71)$ days, that corresponds to indexes over the osteosynthesis with hamate plate $(12.20 \pm 0.97)$ days, Lee hook $(15.10 \pm 0.46)$ days, and percutaneous fixation with wires $(11.70 \pm 0.84)$ days. Thus, the proposed method constitutes an available, less invasive, effective technology that can be recommended for wider use for the surgical treatment of patients with rupture to the $\mathrm{AEC}$ and dislocation of the acromial extremity of the clavicle.

DOI: 10.22448/AMJ.2016.15-16.31-32

UDC 616-001.441

FEATURES OF THE FORENSIC EXAMINATION OF THE DAMAGES BY PIERCING-CUTTING TOOLS

Cheryomkin M.I., Gigolyan M. 0.

Amur State Medical Academy, Blagoveshchensk, Russia

Abstract Determination of the mechanism and conditions for the formation of damages, as well as identification tools of trauma is the main objective of forensics injures from piercing-cutting tools. Bladed weapons, which include primarily various kinds of knives are most often found in the forensic practice. Identification of injuries tools include the definition of a group of blade accessory tools morphologically damage and personal identification. Great significance for the examination of injuries by piercing-cutting tools is taid on the study of the case materials, clinical and primary medical forensic documentation, as well as an experimental research.

Key words: forensic examination, piercing-cutting tools, identification of injury tools.

Piercing-cutting tools - the most common instruments of crime. Bladed weapons are dominated among them, there are different kinds of knives: household designed to perform various kinds of work and knives are steel arms, which were originally made for combat and self-defense. By design features of blades the knives can be divided into two main types: one-sided sharp and two-sided sharp ones. The cross-section with one-sided sharpened knives is usually triangular-wedge shape but two-sided knife blade in cross-section has the form of a narrow elongated diamond [1].

Piercing-cutting damages arise rarely from traumatic action of glass splinters, pieces of iron, daggers, scissors spaced jaws and other subjects which have sharp points and edges.

Damages from the above tools are wounds that are of typical symptoms. The main elements of such damages are wounded channel and input wounded hole - that is actually wound of the skin which has the form of cracks in the closed lumen bounded by two edges and connecting the ends of the wound.

Wounded channel is a slit-like continuation of the inlet into the interior of the body tissues and has a skylight bounded on the side wall. Plane wounded channel walls correspond to wounds closed ends and close up along the lines of edges of the wounded channel, which in its turn in blind wound are connected at an acute angle in the deepest point of the injury - the end of the wounded channel.

The study of the morphology of damages, aimed at defining of a common mechanism and conditions of its formation, the mechanism of formation and dynamics of each feature, allows to select the properties that represent the general structure and characteristics of guns injury, i.e., identification characteristics necessary for identification. Accordingly, there is definition of held group affiliation of the blade piercing-cutting instruments, and in some cases there set a specific instance of the guns in tracks of the blade in the micro-relief of damaged cartilages and bones, if there are any.

The main group features include: the number of blades, the length, width and shape of the blade, the blade features of the base, the shape and thickness of the blade butt.

Determination of the number of blades is set by identifying signs of characteristic of the action's butt of the blade in the butt ends of the wound. Action's butt of the blade on the skin generally causes the formation of rounded or U-shaped end of the wound. Also the depth of the wounded channel in the soft tissue, in the part where the blade butt acted there can be seen connective tissue bridges between the walls of the wounded channel.

Features of the wounded channel by piercing-cutting tools allow to restore the shape of the blade, as well as features such as the presence and shape of the bevel of the blade butt, the nature of the tip and the general 